\title{
ApS en la Educación Superior: La formación práctica de los estudiantes de la Facultad de Ciencias Humanas en el Centro Interdisciplinario de Atención Educativa de la Universidad Autónoma de Baja California
}

\author{
Lilia López Arriaga \\ Universidad Autónoma de Baja California, México \\ Marcela Romero-Jeldres \\ Universidad Metropolitana de Ciencias de la Educación, México
}

\section{Resumen}

El presente artículo relata los procesos históricos de institucionalización del Centro Interdisciplinario de Atención Educativa a la Comunidad (CIAEC) de la Universidad Autónoma de Baja California (UABC) en la ciudad de Mexicali, México, destacando la prospectiva de desarrollo, los antecedentes, los programas y servicios que lo conforma, así como el sustento teórico y metodológico en la formación práctica de los alumnos y de los servicios tranversalizados en el marco del aprendizaje-servicio, después de 29 años atendiendo a la comunidad de Mexicali, y fortaleciendo la formación de los estudiantes de la Facultad de Ciencias Humanas de la UABC. En prospectiva, el CIAEC ha logrado encarnar la Responsabilidad Social Universitaria de la UABC al vincularse con la comunidad y nutrirse del aprendizaje-servicio para gestionar las prácticas profesionales de la formación universitaria, aportando con soluciones comunitarias inclusivas, equitativas, y dotando a los estudiantes de ética y responsabilidad profesional.

\section{Palabras clave}

Aprendizaje-servicio, educación superior, vínculo universidad-comunidad.

Fecha de recepción: 20/IX/2017

Fecha de aceptación: 10/V/2019 


\title{
Service-learning in Higher Education: practical training of students at the Faculty of Human Sciences in the Interdisciplinary Centre for Educational Engagement of the Autonomous University of Baja California
}

\begin{abstract}
This article describes the historical processes of institutionalization of the Interdisciplinary Centre for Educational Engagement with the Community (CIAEC by its Spanish initials) of the Autonomous University of Baja California (UABC) in the city of Mexicali, Mexico, highlighting its development, historical background, and programs and services on offer. This paper also seeks to examine the theoretical and methodological framework underpinning the students' practical training and transversal services that follow a service-learning methodology, after 29 years serving the community of Mexicali, and strengthening the training of students at the Faculty of Human Sciences. The CIAEC has embodied the principles of social responsibility at $U A B C$, by engaging with the community and nurturing service learning to manage professional practices as part of university education, contributing to the community with inclusive, equitable solutions, and providing students with ethical values and professional responsibility.
\end{abstract}

\section{Keywords}

Service-learning, higher education, university-community link. 


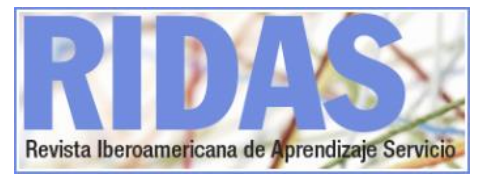

través de la experiencia.

\section{El aprendizaje-servicio como nexo entre la comunidad y las prácticas profesionales}

La UABC establece que su modelo educativo es centrado en el alumno, por tanto, requiere inspirarse en pedagogías activas. En esa idea, Romero (2015) señala que el Aprendizaje-Servicio se nutre de los supuestos teóricos del aprendizaje basado en la experiencia y el servicio a la comunidad y como metodología de intervención comunitaria, orientada a la educación para la ciudadanía, es compatible con otras estrategias educativas planteadas por la UABC, permitiendo cumplir con el criterio de desarrollo de competencias para la vida que se establece en el marco filosófico de la universidad. Al situar a la metodología de aprendizaje-servicio en los programas de la UABC se busca no solo tener una metodología activoparticipativa, sino ir más allá del contexto educativo y dotar a los profesionales en formación que viven la experiencia de aprendizaje de herramientas de desarrollo comunitario, para el ejercicio laboral.

Batlle (2011) y Abston (2015), señalan que países como Holanda, Argentina y Estados Unidos de América, han utilizado esta metodología desde 2009 para desarrollar el concepto de ciudadanía en los alumnos de la educación básica y hasta la educación superior. También ha sido implementada por la American Association of Community Colleges, como una herramienta que mejora los propósitos educativos de los alumnos a
Consecuente con lo anterior, Romero, (2011) señala que para instalar el ApS en las cátedras universitarias, se requiere realizar un proyecto de aprendizaje-servicio, que incluye la perspectiva educativa y social para la solución de problemas en la comunidad, propiciando que el estudiantado desarrolle un proyecto integral que fortalezca a la comunidad. Abston (2015) ratifica lo anterior por cuanto fomenta el desarrollo del capital social de la comunidad fortaleciendo el trabajo en redes, consolidando los valores presentes y sistematiza las normas que aportan cohesión social y contribuye a crear confianza y seguridad entre la población

Ahora bien, desde 1989 la comunidad en el municipio de Mexicali buscaba opciones de atención especializada, es decir, contar con servicios de atención a niños y niñas que presentaban dificultades académicas y de conducta en la escuela. Ello se convirtió en la responsabilidad de un grupo de académicos universitarios de la UABC, guiados por el Mtro. Marco Antonio Villa Vargas, que buscó la participación de profesores de psicología y educación, así como de alumnos que estuvieran interesados en desarrollar y aplicar estrategias viables para la solución de las problemáticas de la comunidad. De modo similar, la iniciativa del Mtro. Marco Antonio Villa Vargas, tiene su correlato con los procesos de formación profesional de los alumnos de las distintas carreras de ciencias sociales y educación de la UABC, las cuales también requerían de un espacio de formación con práctica real 
supervisada.

Así desde 1989 se inicia un programa piloto llamado Centro de Atención Educativa a la Comunidad, como una forma de dar respuesta a las necesidades de la población aledaña a la entonces Escuela de Ciencias de la Educación en Mexicali, Baja California, México. Hoy ese programa es el Centro Interdisciplinario de Atención Educativa a la Comunidad (CIAEC) ubicado dentro la Facultad de Ciencias Humanas.

\section{La institucionalidad del centro}

EI CIAEC está regulado por un acuerdo de creación desde 2005 (Mungaray, 2005; UABC, 2005). Creado como un órgano dependiente de la Facultad de Ciencias Humanas de Mexicali, encargado de coordinar la participación de especialistas en la problemática que se presenta en la comunidad relacionada con temas de salud, capacidades diferentes, familia, comunicación e interacción; diversificar las actividades de práctica académica supervisada y servicios educativos y psicológicos para favorecer el bienestar y el desarrollo de la calidad de vida de la comunidad a través de la vinculación y atención a la misma.

EI CIAEC se conforma bajo una institucionalidad que se organiza dentro de un comité con la participación de un vicerrector del campus Mexicali, un director, un subdirector, coordinadores de área académica (Formación Básica, Formación Profesional y Vinculación Universitaria y Posgrado), docentes y alumnos que por su calidad, méritos y aportaciones al CIAEC, sean nombrados por el rector a propuesta del director.
También establece que a propuesta del director de la Facultad el Rector nombrara un Jefe del Centro. Todo esto para llevar a cabo la planeación y operatividad del Centro, su objetivo y servicios de atención a la comunidad.

Sumado a lo anterior, la oferta de las mejores prácticas de intervención comunitaria se sustenta en la búsqueda de la equidad e inclusión social de aquellas personas que se encuentran en situación de vulnerabilidad. El diseño de las intervenciones de la oferta de servicios, a través de los centros de atención comunitaria y de procesos formativos, también puede unir el servicio social, con las prácticas profesionales. Todo ello está reglamentado en el Estatuto Escolar de la UABC como modalidades no convencionales de obtención de créditos y por las prácticas académicas que se llevan a cabo en las unidades de aprendizaje de los planes de estudio (Mungaray, 2014). No obstante ello, el conocimiento de la Ley de Educación, de la Ley de Salud y del Código Ético de los profesionales involucrados en la atención a la comunidad es la base en la toma de decisiones en cada uno de los programas.

Con el paso de los años, la composición del CIAEC y su proceso de vinculación Universidad-Comunidad se han hecho más complejos. En este momento, para responder a las necesidades de la comunidad en el municipio de Mexicali, que incluye el Valle de Mexicali y San Felipe, el CIAEC además de ejecutar su trabajo con base en la metodología de aprendizaje-servicio, ha debido adoptar el modelo de intervención del Sistema de Salud que se aplica en tres 
dimensiones. Es decir, la dimensión Universal que implica un trabajo de prevención aplicado a la población en general; la dimensión Selectiva, que implica la atención focalizada en individuos y/o grupos con alto riesgo de presentar alguna condición que altere su salud integral y los coloque en situación de vulnerabilidad social; y la dimensión conocida como Indicada, que se enfoca en la atención especializada y especifica de aquellas personas o grupos que requieran un apoyo específico e inmediato (CONADIC, $\mathrm{s} / \mathrm{f}: 8-10$ )

Por lo anterior, la validez social de las intervenciones ha permitido en el tiempo establecer intervenciones viables, deseables y efectivas que eduquen y empoderen a la comunidad, tanto a los futuros profesionistas como a las familias que se atienden. Para ello en el proceso de atención a la comunidad que CIAEC realiza se consideran dos aspectos que dan organización y funcionalidad al centro: uno es el proceso de aprendizaje significativo y colaborativo que se da entre supervisores de servicios, que son profesores universitarios especializados, y los alumnos de los programas educativos de las áreas de Ciencias Sociales (Psicología, Ciencias de la Comunicación, Sociología) y de

Educación y Humanidades (Ciencias de la Educación, Pedagogía); y el otro es la oferta de servicios a la comunidad, operado bajo el modelo de las tres dimensiones buscando brindar opciones de solución a las problemáticas de la comunidad en términos de generación de procesos y grupos que les posibiliten ser autosuficientes. Es aquí donde se ve el impacto de la responsabilidad educativa del CIAEC.

\section{La comunidad}

EI CIAEC trabaja en la comunidad y para la comunidad por lo que la conformación de los programas y servicios de atención se derivan de la solicitud de la comunidad que conoce el trabajo del centro desde hace 27 años. La composición de la población que ha permitido establecer al CIAEC como una institución de formación profesional y de atención comunitaria -reconocido a nivel local- y que recibe la atención en los servicios de CIAEC, es residente del municipio de Mexicali, que incluye a Valle de Mexicali y San Felipe.

Cuadro 1. Distribución de la población que acude a CIAEC en el municipio

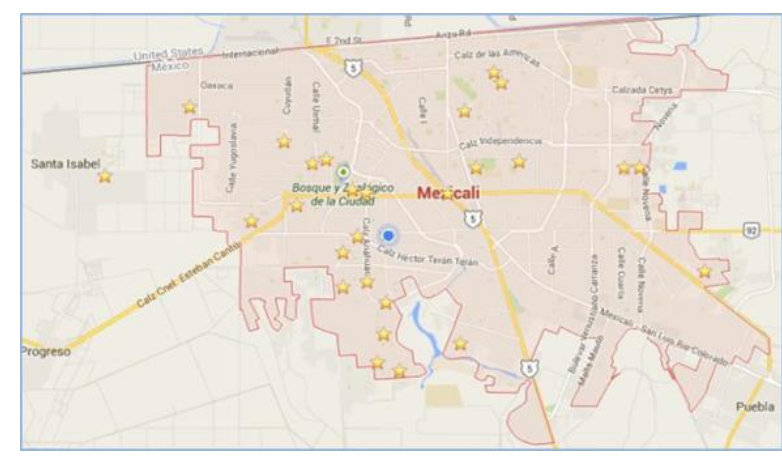

Fuente: Map data (c) Google, INEGI

Las edades de la población atendida fluctúan de entre 18 meses a 50 años, donde el grueso de las personas atendidas está en la edad escolar de educación primaria. La búsqueda de la atención es generalmente debida a que las escuelas refieren los casos y por la recomendación persona a persona. Además, se participa en Brigadas Institucionales, programas de radio y 
televisión.

Durante los últimos 10 años, CIAEC, ha generado espacios de colaboración con otros grupos de población que buscan el apoyo de CIAEC, ya sea en servicios o en capacitación de personal tales como la Alianza de Mujeres en contra de la violencia y las adicciones (16 asociaciones locales), el Colegio de Bachilleres de Baja California, el Instituto Psiquiátrico del Estado de Baja California, la Universidad Nacional Autónoma de México u otras facultades y escuelas de la UABC.

\section{La conformación de los equipos de formación práctica}

En el CIAEC se forman estudiantes de las diferentes licenciaturas de la Facultad de Ciencias Humanas de la UABC en ciencias de la educación, psicología, comunicación y sociología, así como de otras unidades académicas. Provienen del Estado de Baja California, Sonora, Sinaloa y Baja California Sur y realizan sus estudios universitarios en la Universidad Autónoma de Baja California. También CIAEC abre sus puertas a instituciones educativas privadas de educación superior, cuyos estudiantes realizan sus prácticas académicas, de servicio social ${ }^{i}$ comunitario y profesional y voluntariado bajo el acompañamiento de asesores invitados y una continua supervisión.

El equipo académico del CIAEC ha desarrollado un ciclo de formación y capacitación especializada para el estudiante que se integra a los programas y que le permite un tránsito por varias estaciones de desarrollo y crecimiento. Dentro del proceso para formar parte del equipo de CIAEC se considera la participación en una unidad de aprendizaje con carácter de optativa con valor en créditos denominado Psicología Aplicada que se oferta a la comunidad estudiantil de las áreas de educación, ciencias sociales y salud; se lleva a cabo una selección vía entrevista para conocer los intereses e historial académico del alumno que solicita el ingreso a CIAEC y con base en este proceso se realiza la asignación a un equipo.

De este modo, los estudiantes pueden participar a partir del segundo semestre de su carrera, siempre que haya cursado la materia de Psicología Aplicada. Cada semestre se cuenta con la participación de un promedio de 70 estudiantes, entre ellos practicantes, prestadores de servicio social y voluntarios.

Una vez integrados al equipo de trabajo, los equipos de CIAEC realizan capacitaciones previas a la recepción y atención de los estudiantes, así como preparación de materiales de apoyo, cronogramas y procesos de atención. Durante el semestre se continúa la capacitación, se organizan juntas generales y con supervisores, con una formación continua que incluye actividades de revisión de casos, revisión de investigaciones, desarrollo de productos escritos, además de las asesorías y actividades para fomentar la integración grupal y el desarrollo valórico y ético de los integrantes.

Para el desarrollo de estas acciones, se cuenta con el apoyo de profesores de tiempo completo, profesores de 
asignatura y una persona en la administración. Los profesores que participan como asesores de programas y servicios participan con un promedio de 8 horas a la semana, de lunes a viernes en horario de 13 a 16 horas.

\section{Los programas y servicio de CIAEC a la comunidad de Mexicali}

En la actualidad el CIAEC cuenta con el diseño curricular de tres programas de atención, nacidos al alero de las demandas comunitarias y distribuidos en ocho servicios que impactan de manera directa e indirecta a la comunidad, en los que los estudiantes, asesorados por los maestros, practican y desarrollan sus competencias y brindan una atención profesional y servicios de alta calidad.

\subsection{Programa de atención a niños y adolescentes}

Los programas de atención a niños y adolescentes se desglosan en diferentes servicios que incluyen Pediatría Conductual, Desarrollo de Habilidades Sociales, Apoyo Psicopedagógico Infantil, Prevención de Adicciones, Estimulación Temprana y Desarrollo de Habilidades Laborales. En este programa se capacita al estudiante de Psicología, Educación y de la Salud en la evaluación y diagnóstico de las alteraciones leves, moderadas y severas del neuro-desarrollo.

Se habilita al estudiante en el uso de las mejores estrategias que han sido sustentadas en investigación para intervenir en el mejoramiento de las habilidades y aprendizajes de los niños, adolescentes y jóvenes adultos. La intervención se desarrolla con base en una evaluación de la persona que incluye los aspectos contextuales, del desarrollo y conductuales. Se busca la mejor intervención que cumpla con la validez social que corresponde a un trato equitativo y cuyo objetivo es la inclusión social.

Las alteraciones del neuro-desarrollo que se incluyen en estos programas son Trastornos del Espectro Autista, Discapacidad Intelectual y del Desarrollo, Parálisis Cerebral, Déficit de Atención, otras condiciones que requieren atención como: problemas de aprendizaje, problemas en las habilidades de interacción social, discapacidad visual y auditiva y problemas de salud.

\subsection{Programas de atención a adultos y familias}

En estos programas se ofrecen servicios complementarios de apoyo a las familias de los niños y adolescentes que acuden a buscar atención al CIAEC. Se atienden a las familias vía orientación a padres y cuidadores principales y en los casos que se requiera y/o solicite a través de la terapia familiar.

\subsection{Programa de formación y capacitación}

El objetivo general del programa de formación y capacitación busca desarrollar competencias en los alumnos de ciencias sociales, educación y salud en la intervención comunitaria con una metodología de aprendizajeservicio y desde las mejores prácticas fundamentadas en investigación para la solución de problemas reales que 
cumplan los criterios de validez social y responsabilidad social universitaria.

En este programa se incluye el desarrollo de investigaciones que permitan sistematizar y comunicar los procesos formativos y de solución de problemas que se llevan a cabo en la vinculación universidad-comunidad. Además incluye el sistema de atención al público y los procesos comunicativos de CIAEC como organización.

Los procesos de atención en los diferentes programas y servicios se llevan a cabo de acuerdo con el calendario universitario y se planean semestralmente. El trabajo de formación con los estudiantes se inicia en los meses de enero y julio y termina en julio y diciembre de cada año. Los servicios de atención se ofertan de febrero a mayo y de agosto a noviembre de cada año.

Durante 2018 se ha contado con 3 programas y 11 servicios que impactan de manera directa e indirecta a la comunidad, en los que los estudiantes asesorados por los maestros practican y desarrollan sus habilidades brindando una atención profesional y servicios de alta calidad.

\section{Indicadores de seguimiento del plan de desarrollo}

Los indicadores del seguimiento del plan de desarrollo de CIAEC se enmarcan en el plan general de la UABC (2015) y están organizados de acuerdo con parámetros que dan cuenta del cumplimiento de las metas a través de los informes semestrales que realiza CIAEC. Ello se evacua en base a los siguientes informes:

- Presentación de estadísticas y registros de la participación de los alumnos dentro del Centro y las modalidades en las que se desempeñan.

- Asignación de horas/semana/mes para los Profesores de Tiempo Completo y Profesores de Asignatura que participan o puedan participar en el Centro durante el periodo que se desarrolla el presente plan.

- Realización de las capacitaciones establecidas y la asignación de créditos vía modalidades no convencionales (Proyectos de vinculación con valor en créditos, estudios independientes, ayudantías de laboratorio), la elaboración de Convenios de Vinculación y demás modalidades que se establecen dentro de la normatividad universitaria.

- Promoción, aplicación y entrega de certificados de los diferentes Diplomados que se proponen llevar a cabo en el presente plan.

- Presentación de las publicaciones proyectadas en formato de artículo de revista.

- Presentación de proyectos a Convocatorias de Investigación Internas y Externas a la UABC para la consecución de recursos financieros.

- Presentación del trabajo del Centro en las Convocatorias de Financiamiento para programas de 
Servicio Social y otras bolsas que favorezcan la formación profesional de los alumnos de pregrado o licenciatura.

- Realización de estadísticas derivadas de los servicios de atención en cuanto a las dimensiones universal, selectiva e indicada y su alcance valorado por la realización de investigaciones en cuanto a la efectividad de los servicios.

\section{Conclusiones}

La iniciativa académica presentada, es un ejemplo de cómo la Responsabilidad Social Universitaria de la UABC encarna la función social de la universidad como parte del quehacer universitario, al tomar conciencia de sí misma, de su entorno y de su papel dentro de la comunidad de Mexicali. Ello ha implicado diseñar y ofertar un diversificado espectro de programas, proyectos y/o acciones, que en el marco de las funciones institucionales, responde a las necesidades del contexto en el cual está inmersa. Lo anterior, ha demandado por parte de la institucionalidad de la UABC la academia y el estudiantado, un compromiso público con los intereses generales de la comunidad de Mexicali, de la que forma parte, de manera responsable, oportuna, efectiva y con ética.

\section{Referencias bibliográficas}

Abston, G., D. (2015). Outcomes of an Academic Service-Learning Project for Urban Community Colleges. Journal of
Education and Training Studies, 3(3), 61-71.

Batlle, R. (2011). ¿De qué hablamos cuando hablamos de aprendizajeservicio? Crítica, 972, 49-54.

CONADIC (s/f). Modelos Preventivos. Serie Planeación. Consejo Nacional Contra las Adicciones (p. 8-10). Recuperado de http://www.conadic.salud.gob.mx/pdfs/ publicaciones/modprev.pdf

Mungaray, A. (2005). Acuerdo de creación del Centro Interdisciplinario de Atención Educativa a la Comunidad, $U A B C$. Recuperado de https://goo.gl/VskLRc

Mungaray, A. (2014). Estatuto Escolar $U A B C$. Recuperado de https://goo.gl/8QyQPE

Romero, M. (2011). Competencias transversales desde el Aprendizaje y Servicio. Revista Gestión de las Personas y Tecnología, 11, 14-24.

Romero, M. (2015). Ruta estratégica para modernizar CIAEC. Documento de Trabajo en el marco de Movilidad Académica, modalidad Profesor Invitado de la UABC- Facultad de Ciencias Humanas-CIAEC.

i En México, el Servicio Social es un requisito obligatorio para obtener el título profesional, y constituye una obligación jurídica. Tiene una connotación de tipo moral y ético, relacionada con la solidaridad social. 